\title{
SEGURIDAD, SENSIBILIDAD Y APOYO DIFERENCIAL ${ }^{1}$
}

\author{
SAFETY, SENSITIVITY AND DIFFERENTIAL SUPPORT \\ José Zalabardo
}

\begin{abstract}
RESUMEN
En 1999, Sosa sostuvo que el requisito de la sensibilidad no puede recibir apoyo diferencial con respecto al de la seguridad. Esto requiere (D1) que si una creencia que tiene el estatus de conocimiento es sensible, entonces también es segura, y (D2) que si una creencia que no tiene el estatus de conocimiento no es sensible, entonces tampoco es segura. De este modo, el requisito de la seguridad no excluirá de la extensión del conocimiento a ninguna creencia que no deba ser excluida, a menos que el requisito de la sensibilidad lo haga también, y el requisito de la seguridad excluirá de la extensión del conocimiento a toda creencia que deba ser excluida, a menos que el requisito de la sensibilidad no la excluya tampoco. Defiendo que, aunque D1 es correcta, D2 no lo es. Podemos describir creencias verdaderas que no deben ser clasificadas como conocimiento y no satisfacen el requisito de la sensibilidad pero sí el de la seguridad.
\end{abstract}

PALABRAS CLAVE: Ernest Sosa, conocimiento, escepticismo, sensibilidad, seguridad, neo-mooreanismo

\section{SUMMARY}

In 1999, Sosa held that the sensitivity requirement cannot be differentially supported with respect to safety. This in turn requires that (D1) if a belief that has the status of knowledge is sensitive, then it is also safe; and (D2) if a belief that doesn't have the status of knowledge is insensitive, then it is also unsafe. This way, the safety requirement doesn't exclude from the extensión of knowledge any belief that shouldn't be excluded, unless the sensitivity requirement does so too; and the safety requirement excludes from the extensión of knowledge every belief that should be excluded unless the sensitivity requirement doesn't exclude it either. I'm going to

\footnotetext{
${ }^{1}$ Quiero agradecer a Luke Fenton-Glynn, Charlie Neil and Daniel Rothschild sus comentarios sobre este trabajo. Esta investigación ha sido llevada a cabo con el apoyo del Ministerio de Economía y Competitividad del Gobierno de España (proyectos FFI2012-38908-C02-01 and FFI2014-57409). Agradezco este apoyo.
} 
argue that D1 is true, but D2 is false.Some beliefs that should be excluded from the extensión of knowledge fail the sensitivity test but pass the safety test.

KEYWORDS: Ernest Sosa, knowledge, scepticism, sensitivity, safety, neo-mooreanism.

\section{SENSIBILIDAD Y SEGURIDAD}

En "How to Defeat Opposition to Moore", Ernest Sosa atacó la premisa principal de un prometedor argumento escéptico. Según la premisa escéptica que Sosa pretende rechazar, no sé que no soy un cerebro en una cubeta estimulado artificialmente para producir las experiencias de un ser humano normal provisto de un cuerpo, tales como las mías.

Sosa apunta en particular a la línea de apoyo a la premisa escéptica basada en la afirmación de que mi creencia de que no soy un cerebro en una cubeta $(\sim \mathrm{CEC})$ no posee la propiedad que se conoce como sensibilidad:

La creencia de $S$ de que p es sensible precisamente si es el caso que, si p fuera falsa, $S$ no creería que $\mathrm{p}$.

Sosa acepta que mi creencia de que $\sim \mathrm{CEC}$ no es sensible, Pero se opone a la afirmación de que la sensibilidad es una condición necesaria para el conocimiento. Él propone reemplazar la sensibilidad con su contraposición, a la que se refiere como seguridad:

La creencia de $S$ de que $\mathrm{p}$ es segura precisamente si es el caso que, si $\mathrm{S}$ creyera que p, p sería verdadera.

Mi creencia en $\sim$ CEC, sostiene Sosa, es segura. Por lo tanto, reemplazar la sensibilidad con la seguridad como condición necesaria para el conocimiento privaría a la premisa principal del escéptico de esta línea de apoyo.

Sosa bosqueja una línea de razonamiento que, en caso de tener éxito, establecería la conclusión de que "no es posible apoyar diferencialmente a la sensibilidad como el requisito adecuado" (Sosa 1999: 146). De acuerdo con esta conclusión, cada virtud que se pueda asociar con la sensibilidad como condición necesaria para el conocimiento tendrá su contrapartida al conferir este mismo estatus a la seguridad en lugar de la sensibilidad. Mi interés en esta afirmación se limita a sus aspectos extensionales, a la opinión de que la condición de seguridad refleja correctamente la extensión del concepto de conocimiento donde quiera que la condición de sensibilidad también la refleje correctamente. Me referiré a esta tesis 
diciendo que la seguridad domina a la sensibilidad, o como dominación. La dominación puede ser formulada mediante las dos siguientes afirmaciones:

D1 Si una creencia que tiene el estatus de conocimiento es sensible, entonces también es segura.

D2 Si una creencia que no tiene el estatus de conocimiento no es sensible, entonces tampoco es segura.

De acuerdo con D1, el requisito de seguridad no excluye de la extensión del conocimiento a ninguna creencia que no deba ser excluida, a menos que el requisito de sensibilidad lo haga también. De acuerdo con D2, el requisito de seguridad excluye de la extensión del conocimiento a toda creencia que deba ser excluida, a menos que el requisito de sensibilidad no la excluya tampoco. Si D1 y D2 se sostienen, entonces, desde un punto de vista extensional, el requisito de sensibilidad no es mejor que el requisito de seguridad.

Voy a argumentar que D1 es cierto, pero D2 es falso y que, por lo tanto, la dominación es falsa. Hay un aspecto en el que el requisito de sensibilidad es superior al requisito de seguridad. Algunas creencias que deberían ser excluidas de la extensión del conocimiento suspenden el test de la sensibilidad, pero superan el test de la seguridad.

\section{DE LA SENSIBILIDAD A LA SEGURIDAD}

En este apartado quiero abordar la cuestión de si puede haber, a diferencia de D1, instancias de conocimiento que son sensibles pero inseguras. Podemos ver que aspecto tendrían estos casos si traducimos los subjuntivos de sensibilidad y de seguridad al idioma de los mundos posibles. La clave estándar de traducción es la siguiente, donde $\mathrm{p} \rightarrow \mathrm{q}$ representa al condicional subjuntivo si $p$ fuera el caso, entonces q sería el caso:

$\mathrm{K} 1: \mathrm{p} \rightarrow \mathrm{q}$ es verdadera precisamente si en los mundos más cercanos al mundo real en los que p es verdadera, q también es verdad. ${ }^{2}$

Usando esta clave, la sensibilidad es traducida como:

\footnotetext{
${ }^{2}$ La interpretación de la semejanza entre mundos en términos de distancia facilita la exposición. Pueden hacerse afirmaciones similares si adoptamos la interpretación más plausible de esta noción como un ordenamiento parcial. La noción de semejanza entre mundos plantea problemas importantes que podrían socavar su utilidad en la formulación de condiciones para el conocimiento. Véase, por ejemplo, (Kment, 2006). Aquí voy a dejar a un lado estas dificultades.
} 
La creencia de $\mathrm{S}$ de que p es sensible precisamente si, en los mundos más cercanos al mundo real en los que $\mathrm{p}$ es falsa, $\mathrm{S}$ no cree que $\mathrm{p}$.

La aplicación de esta clave de traducción al requisito de seguridad produce un resultado no deseado. Nos da:

La creencia de $\mathrm{S}$ de que $\mathrm{p}$ es segura precisamente si, en los mundos más cercanos al mundo real en los que $S$ cree que $p$, $p$ es verdadera.

Esto no es lo que queremos. Si S cree que p, entonces el mundo más cercano en el que $S$ cree que $\mathrm{p}$ es el mundo real. ${ }^{3}$ Por lo tanto la creencia de $S$ de que $p$ será segura precisamente si es verdadera: toda creencia verdadera será segura. Esto hace que la seguridad sea completamente inefectiva como condición necesaria para el conocimiento.

Sosa sortea este problema utilizando una clave de traducción diferente para la seguridad — una que parece más adecuada para los subjuntivos con antecedentes que son verdaderos-. Requiere que el consecuente del condicional sea verdadero, no sólo en el mundo real, sino también en todos los mundos situados no más allá de una cierta distancia fija d del mundo real en los que el antecedente es verdadero: ${ }^{4}$

$\mathrm{K} 2: \mathrm{p} \rightarrow \mathrm{q}$ es verdadera precisamente si en todo mundo situado a una distancia $\mathrm{d} \mathrm{o}$ menor del mundo real en el que p es verdadera, q también es verdadera.

Utilizando esta clave de traducción, la seguridad se convierte en la siguiente condición no-trivial:

La creencia de $\mathrm{S}$ de que $\mathrm{p}$ es segura precisamente si en todo mundo situado a una distancia d o menor del mundo real en el que $S$ cree que $p$, $p$ es verdadera.

La creencia verdadera de $\mathrm{S}$ de que $\mathrm{p}$ seguirá siendo insegura si existen mundos cercanos (i.e., a una distancia $\mathrm{d}$ o menor) en los que $\mathrm{S}$ cree falsamente que p.

Estamos ya en condiciones de considerar cómo seria una instancia de conocimiento que fuera sensible pero insegura. Tendría que tener como contenido una proposición (verdadera) que es falsa en mundos situados a una distancia menor que d. Para ser sensible debe ocurrir que, en los mundos más cercanos al mundo real en los que $\mathrm{p}$ es falsa, $\mathrm{S}$ no crea $\mathrm{p}$, pero para ser insegura debería haber otros mundos en los que p sea falsa, situados más lejos que los mundos más cercanos en los que $\mathrm{p}$ es falsa, pero aún a una distancia d o menor, en los que $\mathrm{S}$ cree que $\mathrm{p}$.

\footnotetext{
${ }^{3}$ Véase la suposición de centrado fuerte de David Lewis (Lewis 1986b).

${ }^{4}$ Véase la discusión de Robert Nozick en torno a la adherencia (Nozick 1981: 176).
} 
Podemos describir fácilmente creencias verdaderas que posean estos rasgos. Supongamos, por ejemplo, que estoy mirando un jarrón situado sobre una repisa que podría fácilmente no estar ahí, y que la temperatura en la habitación es $18^{\circ}$ centígrados. Supongamos que hay un proyector holográfico enfocado a la repisa y conectado a un termostato, de tal modo que si el jarrón no está sobre la repisa y la temperatura es de $19^{\circ}$ centígrados o superior, proyecta un holograma perfectamente convincente del jarrón. En esta situación mi creencia sería sensible. Asumiendo que la presencia del jarrón no tiene ningún efecto significativo sobre la temperatura de la habitación, en los mundos cercanos en los que no hay un jarrón sobre la repisa no creo que haya un jarrón sobre la repisa, ya que en esos mundos la temperatura es de $18^{\circ}$ centígrados, igual que en el mundo real, y por lo tanto no hay ningún holograma. Sin embargo, existen mundos posibles bastante cercanos —aquellos mundos en los que la temperatura es solamente un poco más alta - en los que creo falsamente que hay un jarrón sobre la repisa, pues soy engañado por el holograma. ${ }^{5}$

Creo que los casos de este tipo son instancias claras de creencias sensibles pero inseguras, tal como han sido formuladas estas nociones. ${ }^{6}$ Sin embargo, por sí solo esto no los convierte en contraejemplos a D1. Para ello se necesitaría, además, que podamos reconocer intuitivamente estos casos como instancias de conocimiento. En este respecto, los ejemplos no dan la talla. Me parece que en estos casos nuestras intuiciones son contradictorias, y no lo suficientemente robustas para decidir la suerte de D1.

Quiero sugerir, no obstante, que esta cuestión es irrelevante, ya que la clase de casos considerada es posible únicamente gracias a una política de traducción de subjuntivos al lenguaje de los mundos posibles muy cuestionable. El problema afecta a la decisión de utilizar diferentes claves de traducción para la sensibilidad y la seguridad. Esto podría ser justificado únicamente sobre la base de que, en los casos que nos interesan (los de creencia verdadera), el antecedente del subjuntivo de sensibilidad será falso pero el antecedente del subjuntivo de seguridad será ver-

\footnotetext{
${ }^{5}$ Para que los mundos más cercanos en los que no hay un jarrón sobre la repisa y la temperatura es $19^{\circ}$ centígrados estén más alejados del mundo real que los mundos más cercanos en los que no hay un jarrón en la repisa pero la temperatura es de $18^{\circ}$ centígrados, la semejanza aproximada en hechos particulares tiene que tener alguna relevancia en la relación de semejanza entre mundos. Los criterios de Lewis permiten que sea así, si bien no lo requieren (Lewis 1986a: 4748). Podríamos describir fácilmente ejemplos que no están sujetos a esta condición.

${ }^{6}$ Esto es así en presupuesto plausible de que la distancia a la que están del mundo real los mundos más cercanos en los que no hay un jarrón sobre la repisa y la temperatura es $19^{\circ}$ centígrados no es mayor que d.
} 
dadero. Pero parece erróneo hacer que las condiciones de verdad de un subjuntivo dependan del valor de verdad de su antecedente. Consideremos, por ejemplo, el siguiente subjuntivo, "si la moneda fuera devaluada, los tipos de interés subirían". De acuerdo con la propuesta que estamos discutiendo, si la moneda es realmente devaluada, la verdad del subjuntivo requeriría aumentos de los tipos de interés, no solo en la realidad, sino también en el rango de condiciones existentes en todos los mundos situados a una distancia d o menor en los que la moneda es devaluada, por ejemplo, con una variedad de niveles impositivos, inflación, etc., pero si la moneda no es devaluada, para que el subjuntivo sea verdadero será suficiente que los tipos de interés suban en las circunstancias específicas existentes en los mundos más cercanos en los que la moneda es devaluada, por ejemplo, con los niveles precisos de imposición, inflación, etc. presentes en esos mundos. Creo que esto es contraintuitivo. Si, para evitar que la seguridad sea redundante, tenemos en cuenta un rango más amplio de mundos para determinar el valor de verdad del subjuntivo, necesitamos aplicar la misma estrategia cuando se trata de la sensibilidad.

Para toda proposición contingente $\mathrm{p}$, digamos que $\mathrm{CT}(\mathrm{p})$ denota la distancia a la que está del mundo real el mundo más cercano en el que p es verdadera. Con la ayuda de esta función podemos formular fácilmente la clave de traducción que proporciona los resultados deseados:

K3: $\mathrm{p} \rightarrow \mathrm{q}$ es verdadera precisamente en el caso de que en todo mundo situado a una distancia $\mathrm{CT}(\mathrm{p})+\mathrm{d}$ o menor del mundo real en el que $\mathrm{p}$ es verdadera, $\mathrm{q}$ también es verdadera. ${ }^{\top}$

Para subjuntivos con antecedentes verdaderos, K3 proporciona la misma traducción que $\mathrm{K} 2$, ya que $\mathrm{CT}(\mathrm{p})=0$ siempre que $\mathrm{p}$ sea verdadera. Por lo tanto la noción de seguridad para creencias realmente existentes sigue siendo la misma. Sin embargo, para subjuntivos con antecedentes falsos, las traducciones generadas por K3 difieren de aquellas generadas por K1. La sensibilidad se formula ahora de la siguiente manera:

La creencia de $\mathrm{S}$ de que $\mathrm{p}$ es sensible precisamente si en todo mundo situado a una distancia de $\mathrm{CT}(\sim \mathrm{p})+\mathrm{d}$ del mundo real en el que $\mathrm{p}$ es falsa, $\mathrm{S}$ no cree que $\mathrm{p} .{ }^{8}$

\footnotetext{
${ }^{7}$ Véase la discusión de Robert Nozick en torno a la adherencia (Nozick 1981: 176).

8 Repárese en que esta definición no tiene aplicación en el caso de las proposiciones necesariamente verdaderas. Si p es necesariamente verdadera, entonces CT no proporciona ningún valor para $\sim$ p. Esto explica por qué, como observó Nozick, la restricción de sensibilidad no excluye de la extensión del conocimiento a ninguna creencia en una verdad necesaria (Nozick 1981: 186).
} 
En esta interpretación, para que la creencia de $S$ que p sea sensible, $S$ tiene que abstenerse de creer que $\mathrm{p}$, no solo en los mundos posibles más cercanos en los que p es falsa, sino también en cualquier otro mundo en que p sea falsa a una distancia del mundo real que exceda la de éstos en d o menos. ${ }^{9}$ En esta interpretación, la sensibilidad es más difícil de lograr.

Estoy afirmando que esta es la interpretación que tenemos que utilizar a la hora de considerar la relación entre seguridad y sensibilidad. ${ }^{10} \mathrm{Y}$ en esta interpretación no hay margen para contraejemplos a D1, ya que la sensibilidad es ahora estrictamente más fuerte que la seguridad.

Para verlo ayudará que consideremos que la sensibilidad y la seguridad son propiedades que descartan mundos posibles cercanos en los que $\mathrm{S}$ cree falsamente que p, i.e. mundos en los que $S$ cree que $p$, pero en los que p no es el caso. Llamemos a estos mundos mundos de error. Tanto la sensibilidad como la seguridad imponen un límite inferior a la distancia del mundo real en que podemos encontrar mundos de error. La única diferencia entre las dos propiedades es que, mientras que el límite inferior impuesto por la sensibilidad puede variar junto con la proposición creída, la seguridad impone un límite inferior fijo —el mismo para cualquier proposición-. Por un lado, la creencia de S que p es sensible precisamente si el mundo de error más cercano se encuentra a una distancia mayor que $\mathrm{CT}(\mathrm{p})+\mathrm{d}$. Por otro lado, la creencia de $\mathrm{S}$ que p es segura precisamente si el mundo de error más cercano se encuentra a una distancia mayor que d.

En esta forma de ver las cosas, es fácil ver por qué la sensibilidad es más fuerte que la seguridad. CT únicamente produce valores no negativos. Por lo tanto para cualquier proposición $\mathrm{p}, \mathrm{CT}(\mathrm{p})+\mathrm{d} \geq \mathrm{d}$. Por lo tanto, si no existen mundos de error a una distancia de $\mathrm{CT}(\mathrm{p})+\mathrm{d}$ o menor, como requiere la sensibilidad, no existirá ningún mundo de error a una distancia de $\mathrm{d}$ o menor, como requiere la seguridad. Podemos concluir que toda creencia sensible es también segura.

Esto destruye cualquier esperanza de encontrar contraejemplos a D1. Tendrían que ser creencias con el estatus de conocimiento que son sensibles pero no

\footnotetext{
${ }^{9}$ Creo que esta era la intención original de Nozick. Véase (Nozick 1981: 176).

${ }^{10}$ Lo que afirmo es que esta es la mejor interpretación para los subjuntivos que son ampliamente utilizados para formular la sensibilidad y la seguridad. Sin embargo, como Sherrilyn Roush ha mostrado, estas nociones también pueden ser formuladas en términos de probabilidades condicionales (Roush 2005). He defendido esta estrategia en (Zalabardo 2012). Para una discusión sobre la relación entre la sensibilidad y la seguridad (y la adherencia) en un marco probabilístico, véase (Zalabardo 2012: 115-18).
} 
seguras. No puede haber ninguna de éstas porque toda creencia sensible, con o sin el estatus de conocimiento, es también segura. ${ }^{11}$

\section{DE LA SEGURIDAD A LA SENSIBILIDAD}

Pasemos a D2 - el principio según el cual toda creencia verdadera insensible que no tenga el estatus de conocimiento también es insegura-. Nuestra pregunta es si existen contraejemplos a este principio — creencias verdaderas seguras pero insensibles a las que no debemos conceder el estatus de conocimiento-. Voy a argumentar que casos de este tipo pueden describirse fácilmente. Para verlo, reparemos en que lo que se requiere para la seguridad de una creencia en una proposición verdadera p cambia drásticamente dependiendo de si CT $(\sim \mathrm{p})$ excede d. Cuando $\mathrm{CT}(\sim \mathrm{p}) \leq \mathrm{d}$, las disposiciones doxásticas de $\mathrm{S}$ tienen que ser tales que en los mundos a un distancia $\mathrm{d}$ o menor del mundo real en los que $\mathrm{p}$ es falsa, $\mathrm{S}$ no cree que $\mathrm{p}$. Si lo creyera existirían mundos erróneos a una distancia d o menor del mundo real, y la creencia de S sería insegura. No obstante, cuando $\mathrm{CT}(\sim \mathrm{p})>$ d, la seguridad no exige nada a las disposiciones doxásticas de $\mathrm{S}$. Si no existen mundos posibles a una distancia d o menor del mundo real en los que p es falsa, entonces no hay, a fortiori, mundos de error a una distancia d o menor del mundo real. Por lo tanto, cualquier creencia en p será segura, independientemente de las disposiciones doxásticas del sujeto con respecto a p y, específicamente, independientemente de si hacen que su creencia sea sensible. ${ }^{12}$ Pues la sensibilidad, a diferencia de la seguridad, impone exigencias a las disposiciones doxásticas del sujeto independientemente del valor de $\mathrm{CT}(\sim \mathrm{p})$. Independientemente de lo alto que pueda ser este valor, para que la creencia de $\mathrm{S}$ en $\mathrm{p}$ sea sensible, sus disposiciones doxásticas respecto a $\mathrm{p}$ tendrán que ser tales que impidan la creencia en $\mathrm{p}$ en algunos mundos, i.e. en los mundos situados a una distancia $\mathrm{CT}(\sim \mathrm{p})+\mathrm{d}$ o menor del mundo real en los que $\mathrm{p}$ es falsa.

Algunas consecuencias de esta disparidad son bienvenidas por Sosa. Esta es la razón por la que el requisito de sensibilidad excluye mi creencia en $\sim$ CEC de la

\footnotetext{
${ }^{11}$ El resultado que importa para nuestros propósitos es que, a este respecto, la seguridad no es peor que la sensibilidad. Cada instancia de conocimiento que pasa la prueba de sensibilidad también pasará la prueba de seguridad. Sin embargo, también hemos establecido que la seguridad no es mejor que la sensibilidad. Cualquier creencia verdadera a la que no deba serle concedido el estatus de conocimiento pero pasa la prueba de sensibilidad también pasará la prueba de seguridad.

12 En (Zalabardo 2009) introduje la noción de libertad del riesgo de error, que permite una transición gradual entre las dos maneras de satisfacer la seguridad.
} 
extensión del conocimiento, pero la seguridad no lo hace. La gran distancia con respecto al mundo real de los mundos CEC más cercanos —-más allá de cualquier valor plausible que podamos asignar a d — significa que mi creencia en $\sim \mathrm{CEC}-\mathrm{y}$, de hecho, cualquier creencia en esta proposición por parte de un sujeto encarnado en el tipo de mundo que creemos que habitamos - será segura, independientemente de mis disposiciones doxásticas con respecto a esta proposición. La sensibilidad, por el contrario, impone demandas que no son satisfechas por mis disposiciones doxásticas en relación a $\sim$ CEC. Esta es la razón por la que reemplazar la sensibilidad con la seguridad como condición necesaria para el conocimiento abre la posibilidad de adoptar la respuesta mooreana al argumento escéptico que Sosa recomienda.

Si, como mantiene Sosa, sé que no soy un cerebro en una cubeta, esta creencia segura pero insensible mía no es un contraejemplo a D2.. ${ }^{13}$ Quiero argumentar, sin embargo, que otras creencias que son seguras pero insensibles no pueden ser plausiblemente consideradas instancias de conocimiento.

Un contraejemplo a D2 sería un caso en el que un sujeto S cree una proposición verdadera p y se dan las siguientes condiciones:

1. $\mathrm{S}$ no sabe que $\mathrm{p}$

2. La creencia de $S$ que $p$ no es sensible.

3. La creencia de $S$ que $\mathrm{p}$ es segura.

Un caso de este tipo tendría que ser excluido de la extensión del conocimiento por una condición necesaria para la instanciación del concepto, y mientras la condición de sensibilidad lograría excluirlo, la condición de seguridad no lo lograría.

Como señalamos hace un momento, una condición suficiente para que la creencia de $\mathrm{S}$ de que p sea segura es que $\mathrm{CT}(\sim \mathrm{p})$ sea mayor que d. Tendríamos, por lo tanto un contraejemplo a D2 si pudiéramos encontrar un caso que satisficiera las condiciones 1 y 2 anteriores, además de:

$$
\text { 3*. } \mathrm{CT}(\sim \mathrm{p})>\mathrm{d}
$$

Sosa dice muy poco acerca de la extensión de la esfera-d. Todo lo que sabemos es que incluye mundos que fácilmente podrían haber sido reales, y que excluye mundos los CEC — los mundos en los que soy un cerebro en una cubeta—. Por lo tanto la única manera en la que podemos estar seguros de que una proposición $\mathrm{p}$ satisface la condición $3^{*}$ es si los mundos más cercanos en los que es falsa no están

\footnotetext{
${ }^{13}$ Coincido con Sosa en este punto, aunque por razones diferentes a las suyas. Véase (Zalabardo 2012: 136-39).
} 
más cercanos al mundo real que los mundos CEC. Pero esto es todo lo que se necesita para generar contraejemplos a D2. Consideremos la siguiente proposición:

CEC6: El MI6 mantiene secretamente una colección de cerebros en cubetas artificialmente estimulados para producir las experiencias de un ser humano normal encarnado.

Asumamos que CEC6 es falso. Afirmo que Sosa no puede negar que CT (CEC6) > d, pues los mundos en los que el MI6 mantiene una colección de cerebros en cubetas no están situados más cerca del mundo real que los mundos en los que yo soy un cerebro en una cubeta. De acuerdo con cualquier medida plausible de la semejanza, los primeros son al menos tan diferentes del mundo real como los segundos. Por supuesto, algunos mundos CEC difieren del mundo real en aspectos bastante radicales no compartidos por los mundos CEC6. Sin embargo, habrá también mundos CEC que son bastante semejantes al mundo real, excepto por el hecho de que aquellos contienen cerebros encubetados. Sin duda Sosa desearía situar estos mundos CEC a una distancia mayor que d ya que, de otra manera no superarían el test de la seguridad, y el cambio de la sensibilidad a la seguridad sería de poca ayuda contra el escéptico. Pero esta diferencia también está presente en los mundos CEC6. No alcanzo a ver por qué el hecho de que en los mundos CEC la víctima de este procedimiento sea yo deba situar a estos mundos más lejos que los mundos CEC6, en los que otros sufren esta suerte. Se sigue de aquí que cualquier creencia en $\sim$ CEC6 satisfará la condición $3^{*}$ y, a fortiori, que será segura. Por lo tanto, para obtener un contraejemplo a D2 bastará con encontrar una creencia en $\sim$ CEC6 que satisfaga las condiciones 1 y 2.

$\sim$ CEC6 es perfectamente cognoscible. Alguien con el nivel apropiado de acceso sería capaz de conocer $\sim$ CEC6. E incluso sin esto, alguien con el conocimiento correcto de lo que es técnicamente posible en este área sería capaz de conocer $\sim$ CEC6. Es plausible incluso decir que la mayoría de nosotros conoce $\sim$ CEC6 de esta manera: sé que el MI6 no colecciona cerebros en cubetas porque es técnicamente imposible hacer tal cosa. Pero, ¿podemos decir que cualquiera que crea CEC6 los sabe? Voy a argumentar que esta pregunta debería ser respondida negativamente.

Consideremos a Roger, quien cree $\sim$ CEC6, pero por razones bastante poco ortodoxas. Roger no cree que sea técnicamente imposible mantener cerebros en cubetas. De hecho, cree que es algo que ocurre con regularidad, pues ha oído hablar del experimento mental de Putnam y se formó una idea errónea de la situación. Sin embargo, está convencido de que el MI6 no se implica en este tipo de actividades, y la razón es que tiene un amigo que le dice que trabaja para el MI6 y 
que siempre está dispuesto a responder a sus preguntas sobre la organización. En realidad, el amigo de Roger pertenece al servicio de limpieza de la sede del MI6, no tiene ningún acceso a información clasificada, y proporciona respuestas aleatorias pero coherentes a las preguntas que le hace Roger únicamente para complacerle. Cuando Roger le preguntó si el MI6 coleccionaba cerebros en cubetas, le aseguró que no lo hacía. Es por estos motivos que Roger cree $\sim$ CEC6.

Me parece obvio que la creencia verdadera de Roger en $\sim$ CEC6 no posee el estatus de conocimiento y, por lo tanto, que satisface la condición 1. El testimonio del amigo de Roger acerca de las actividades del MI6 no es una fuente de conocimiento, y Roger no conoce ninguna de las verdades en las que llega a creer por medio de este testimonio.

Por lo tanto la creencia verdadera de Roger debería ser excluida de la extensión del conocimiento, pero la condición de seguridad no logra hacerlo, ya que la creencia de Roger, como cualquier otra creencia en $\sim$ CEC6, es segura. Todo lo que necesitamos mostrar ahora para obtener un contraejemplo a D2, es que la condición de sensibilidad tiene éxito allí donde la condición de seguridad fracasa, i.e. que la creencia de Roger es insensible.

Esto puede mostrarse fácilmente. Consideremos los mundos posibles más cercanos (hasta una distancia de CT(CEC6) + d) en los que el MI6 mantiene una colección de cerebros en cubetas. Podemos esperar que en al menos algunos de estos mundos el amigo de Roger sigue diciendo que el MI6 no colecciona cerebros en cubetas, y Roger sigue creyendo en lo que su amigo le dice. Se sigue que la creencia real de Roger en $\sim$ CEC6 es insensible, como pretendíamos mostrar.

En conclusión, la creencia de Roger en $\sim \mathrm{CEC6}$ es una creencia verdadera segura pero insensible a la que no deberíamos conceder el estatus de conocimiento. Se sigue que tenemos un contraejemplo a D2: algunas de las creencias verdaderas que deberían ser excluidas de la extensión del conocimiento son excluidas por la condición de sensibilidad pero no por la condición de seguridad. Concluyo que la afirmación de Sosa de que la seguridad domina a la sensibilidad debería ser rechazada. Obsérvese, sin embargo, que esta conclusión no invalida la concepción del conocimiento de Sosa basada en la seguridad. Sosa nunca ha afirmado que la seguridad sea la única condición necesaria para el conocimiento (i.e., una condición suficiente), y la creencia de Roger sin duda será excluida de la extensión del conocimiento por otras condiciones que Sosa contempla. ${ }^{14} \mathrm{Mi}$ afirmación es mucho

\footnotetext{
${ }^{14}$ Tampoco estoy diciendo que la sensibilidad emerja victoriosa de su enfrentamiento con la seguridad. Puede muy bien ser que la condición de sensibilidad represente erróneamente la
} 
más modesta. La concepción global de Sosa podría muy bien ser capaz de excluir de la extensión del conocimiento todas las creencias que serían correctamente excluidas por el requisito de sensibilidad, pero contrariamente a lo que Sosa afirmó en 1999, el requisito de seguridad no puede hacerlo por sí solo. La sensibilidad puede recibir apoyo diferencial como el requisito correcto.

Traducción de David Pérez Chico

$$
\begin{array}{r}
\text { José Zalabardo } \\
\text { University College London } \\
\text { j.zalabardo@ucl.ac.uk }
\end{array}
$$

\section{BIBLIOGRAFÍA}

KMENT, Boris (2006), "Counterfactuals and Explanation”. Mind 115:261-310.

LEWIS, David (1986a), "Counterfactual Dependence and Time's Arrow”. En Philosophical Papers. Volumen II. Oxford: Oxford University Press.

- (1986b), "Counterfactuals and Comparative Possibility". En Philosophical Papers. Volumen

II. Oxford: Oxford University Press.

NozICK, Robert (1981), Philosopbical Explanations. Cambridge, Massachusetts: Harvard University Press.

RousH, Sherrilyn (2005), Tracking Truth. Oxford: Oxford University Press.

SosA, Ernest (1999) "How to Defeat Opposition to Moore". En Pbilosophical Perspectives,

13, Epistemology, editado por J. E. Tomberlin. Malden, Massachusetts y Oxford: Blackwell.

Zalabardo, José L. (2009), "How I Know I'm Not a Brain in a Vat". Royal Institute of Philosophy Supplement 64:65-88. Versión espannola: "Cómo sé que no soy un cerebro en una cubeta”. En Conocimiento y Escepticismo. Ensayos de Epistemología. México DF: Instituto de Investigaciones Filosóficas, 2014.

— (2012) Scepticism and Reliable Belief. Oxford: Oxford University Press.

extensión del conocimiento en aspectos más importantes que el tipo de problema planteado por creencias como la de Roger para la condición de seguridad. 